\title{
The structures and proteolytic specificities of autolysed human thrombin
}

\author{
Jui-Yoa CHANG \\ Pharmaceuticals Research Laboratories, CIBA-GEIGY Ltd., Basel CH-4002, Switzerland
}

\begin{abstract}
Three Arg/Lys-Xaa bonds in the B-chain of human $\alpha$-thrombin were found to be the major autolytic sites. Under the conditions of $1 \mathrm{mg}$ of $\alpha$-thrombin/ml in $50 \mathrm{~mm}$-ammonium bicarbonate solution at $25^{\circ} \mathrm{C}$, the $50 \%$ cleavage times of Lys-Gly (residues 154-155), Arg-Tyr (residues 70-71) and Arg-Asn (residues 73-74) were $32 \mathrm{~h}, 72 \mathrm{~h}$ and $96 \mathrm{~h}$ respectively. Fragments generated from these three major autolytic sites were purified and analysed. In addition, minor and random autolytic cleavages occurred simultaneously that eventually led to the complete breakdown of the enzyme. These results reveal several novel aspects about the process of autolysis and the structure of autolysed human thrombin. (1) It identifies a major autolytic site at Arg-Tyr (residues 70-71) that has not been previously reported. (2) It demonstrates that $\beta$-thrombin is not an obligatory intermediate during the process of conversion of $\alpha$-thrombin into $\gamma$-thrombin. There exists a new form of autolysed thrombin, designated as $\beta^{\prime}$-thrombin (with cleavage at Lys-Gly only), which also serves as the intermediate in the conversion of $\alpha$-thrombin into $\gamma$-thrombin. (3) It shows that autolysis of human $\alpha$-thrombin does not proceed in an absolutely clear-cut manner. Numerous minor cleavages, which amount to approx. $20 \%$ of the three major autolytic sites, occur simultaneously. (4) It is the first time that several autolytic sites of human $\alpha$-thrombin have been quantitatively analysed, and that it has been shown that formation of $\beta$-, $\beta^{\prime}$ - and $\gamma$-thrombins can be quantitatively followed by the h.p.l.c. method. Furthermore, the data demonstrate that $\alpha$-thrombin and the autolysed thrombin (mixture of $\beta-, \beta^{\prime}$ - and $\gamma$-thrombins) have comparable proteolytic activity and specificity towards various sizes of non-fibrinogen polypeptide substrates with relative molecular masses ranging from 3000 to 25000 .
\end{abstract}

\section{INTRODUCTION}

Human $\alpha$-thrombin is an important bioregulatory serine proteinase that catalyses the proteolytic conversion of fibrinogen into fibrin (Magnusson, 1972; Lundblad et al., 1976; Fenton et al., 1978; Fenton, 1981). This reaction involves the selective cleavage of two different Arg-Gly bonds from a total of $181 \mathrm{Arg} / \mathrm{Lys}-\mathrm{Xaa}$ bonds within fibrinogen (Blombäck et al., 1967). The exact molecular mechanism that governs this specificity is only partially understood (Blombäck et al., 1967; Hogg \& Blombäck, 1978; Fenton et al., 1978; Marsh et al., 1983; Chang, 1983a, 1985; Hanna et al., 1984). There are three forms $(\alpha, \beta$ and $\gamma)$ of thrombin (Rosenberg \& Waugh, 1970; Glover \& Shaw, 1971; Gorman et al., 1976; Fenton et al., 1978; Lundblad et al., 1979). Only the naturally occurring $\alpha$-thrombin possesses the clotting activity. Both $\beta$ - and $\gamma$-thrombins are derived from $\alpha$-thrombin through autolysis or limited proteolysis by trypsin and similar enzymes (Fenton et al., 1977a, 1978). One interesting property of both $\beta$ - and $\gamma$-thrombins is that they still retain substantial proteolytic activity towards small tripeptide substrates (Magnusson, 1972; Lundblad et al., 1976; Fenton et al., 1978; Fenton, 1981). This suggests that the active site of $\alpha$-thrombin remains essentially intact within both $\beta$ - and $\gamma$-thrombins. Thus structural analysis of $\beta$ - and $\gamma$-thrombins and characterization of their proteolytic activities may provide an insight into the mechanism that accounts for the specific clotting activity of $\alpha$-thrombin.

The structures of autolysed bovine (Lundblad et al., 1979) and human (Fenton et al., 1977a; Boissel et al., 1984) $\alpha$-thrombins have been reported. However, quantitative data concerning the autolytic cleavages are not available. The aim of the present study was to establish the relative cleavage rates at each of the $\alpha$-thrombin autolytic sites and to compare the proteolytic specificities of $\alpha$-thrombin and its autolysed derivatives towards non-fibrinogen substrates.

\section{MATERIALS AND METHODS}

\section{Materials}

Human $\alpha$-thrombin (2500 NIH units/mg) was kindly provided by Dr J. W. Fenton, II (Albany, NY, U.S.A.). The purity of $\alpha$-thrombin was ascertained by SDS/polyacrylamide-gel electrophoresis, quantitative $N$-terminal analysis and h.p.l.c. and was found to contain at least $98 \%$ of the $\alpha$-form. Dimethylaminoazobenzene isothiocyanate and dimethylaminoazobenzenesulphonyl chloride were products of Fluka (Buchs, Switzerland). Other chemicals and solvents used in this study were commercial analytical grade from either Merck or Fluka. Chromozym TH (tosyl-Gly-Pro-Arg-4-nitroanilide acetate) was from Boehringer Mannheim. Antibody $\kappa$-chain $10 \mathrm{~K} 26$ was isolated as described previously (Alkan et al., 1983; Chang et al., 1985). Gastrin-releasing peptide, calcitonins and fibrinogenderived peptide $\mathrm{A} \alpha(1-23)$ were obtained from Bachem (Bubendorf, Switzerland).

\section{Autolysis of human a-thrombin}

This was carried out in $50 \mathrm{~mm}$-ammonium bicarbonate, $\mathrm{pH} 8$, at $25^{\circ} \mathrm{C}$ with the concentration $1 \mathrm{mg} / \mathrm{ml}$. At time intervals, autolysed samples were re- 
moved for direct analysis of their clotting activities and proteolytic activities, or were removed, freeze-dried and used for (a) quantitative $N$-terminal analysis, (b) SDS/ polyacrylamide-gel electrophoresis, (c) gel-filtration (Sephadex G-75) separation and (d) h.p.l.c. analysis.

\section{Activity assay of thrombin derivatives}

Clotting activity was measured by a Coagulometer KC-1 (Merz and Data, Düdingen, Switzerland) with human fibrinogen (Kabi) as substrate. Proteolytic activities on non-fibrinogen polypeptides were analysed with the conditions described in Chang (1985).

\section{Structure characterization of polypeptides}

Quantitative $N$-terminal analysis was performed by the dimethylaminoazobenzene isothiocyanate method (Chang, 1983b,c). Amino acid composition was determined by the dimethylaminoazobenzenesulphonyl chloride method (Chang et al., 1983). Sequence of polypeptides was determined by both the dimethylaminoazobenzene isothiocyanate/phenyl isothiocyanate method (Chang, 1983c) and gas-phase Sequenator (Hunkapiller et al., 1983). Glass filters were loaded with $5 \mathrm{mg}$ of Polybrene and precycled five times before $0.3-1.5 \mathrm{nmol}$ of polypeptides was applied. Dimethylaminoazobenzenethiohydantoin derivatives (Chang, 1983c) and phenylthiohydantoin derivatives (Knecht et al., 1983) of amino acids were quantitatively analysed by h.p.l.c. SDS/polyacrylamide-gel electrophoresis was performed by the method of Laemmli (1970).

\section{RESULTS}

\section{Characterization of the structures of autolysed human a-thrombin}

To ensure detection of every major and minor cleavage site during autolysis of $\alpha$-thrombin, autolysed samples were analysed by quantitative $N$-terminal analysis before any chromatographic separations. Portions of autolysed $\alpha$-thrombin were removed at time intervals and freeze-dried. The newly appeared $N$-termini were determined by the dimethylaminoazobenzene isothiocyanate method. In addition to the $N$-termini of A-chain (Thr) and B-chain (Ile), three major new $\mathrm{N}$-termini, Gly, Tyr and Asn, appeared increasingly as autodigestion continued (Fig. 1). There were also minor new $N$-termini, notably Ser, Ile, Asp and His. When autolysis was allowed to proceed longer than $144 \mathrm{~h}$, $N$-terminal analysis indicated that random and total fragmentation started to prevail, as virtually all amino acid dimethylaminoazobenzenethiohydantoin derivatives increased.

On SDS/polyacrylamide-gel electrophoresis under non-reducing conditions, accompanying the decrease of $\alpha$-thrombin, two well-separated groups of components with $M_{\mathrm{r}}$ values 27000 and 14000 (Fenton et al., 1977a) appeared to increase (Fig. 2). Each of these components was subsequently isolated by gel filtration (Fig. 3) and h.p.l.c. (Fig. 4) from $144 \mathrm{~h}$-autolysed $\alpha$-thrombin and their structures were determined by amino acid sequencing and amino acid composition analysis. Their structures are summarized in Table 1 and Fig. 5. For fragments A-B2 and A-B5, each component is actually comprised of two molecules with three amino acid residues differing at the $N$-terminal end of the B-chain (Table 1). In Fig. 2, fragment A-B5 was resolved into two

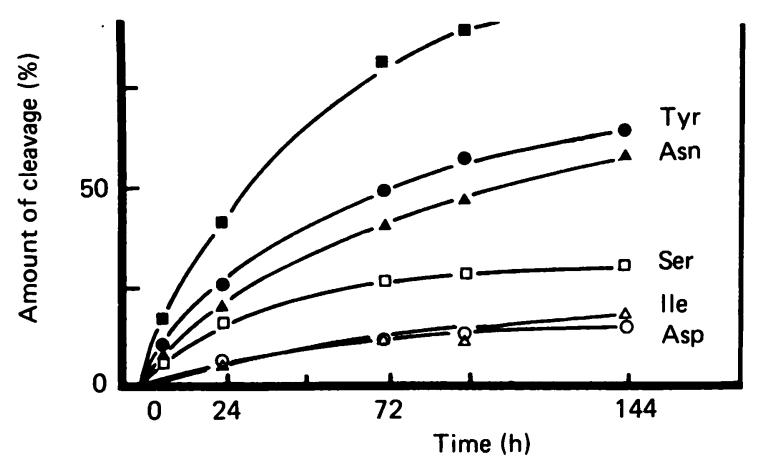

Fig. 1. Quantitative $N$-terminal analysis of autolysed human $\alpha$-thrombin by the dimethylaminoazobenzene isothiocyanate method

Human $\alpha$-thrombin has two $N$-termini, from A-chain (Thr) and B-chain (Ile). The recoveries of newly appeared $N$-termini were quantitatively analysed and 'amount of cleavage (\%)' was defined as the recoveries of new $\mathrm{N}$-termini relative to the $\mathrm{N}$-terminus of A-chain (Thr) taken as $100 \%$. Recoveries of new $N$-termini with less than $15 \%$ after $144 \mathrm{~h}$ autolysis are not shown. The recovery of His (about $15-25 \%$ after $144 \mathrm{~h}$ ) is not shown because of the difficulty of evaluating the histidine dimethylaminoazobenzenethiohydantoin derivative. New Gly, Tyr and Asn $N$-termini are formed as shown in Table 1. The $N$-terminal Ser was derived from minor autolytic cleavage at Lys-Ser (212-213) as discussed in the text. The origin of Asp is unknown.

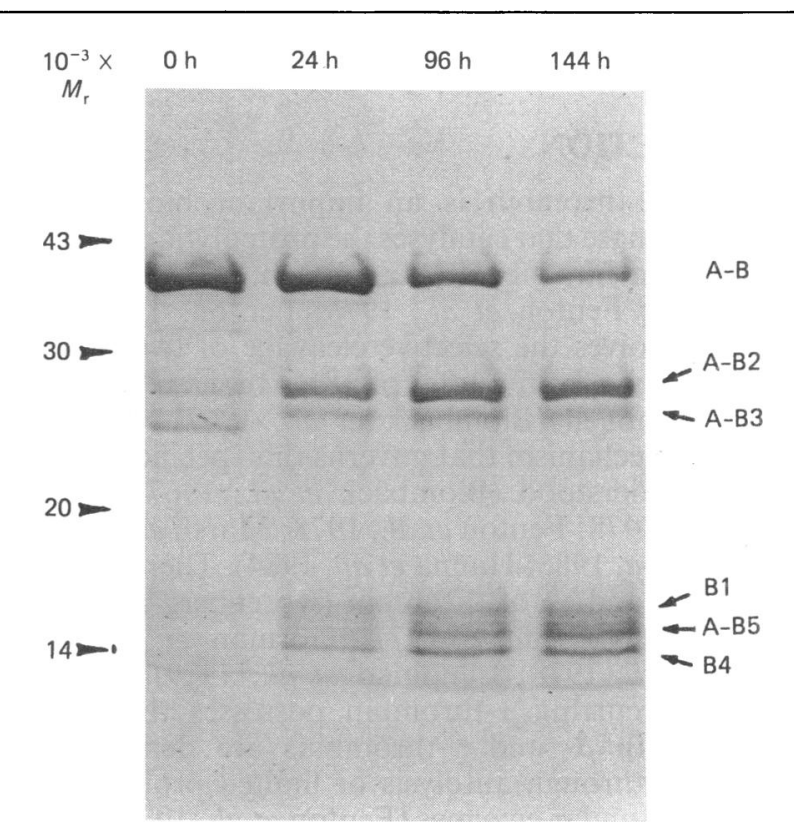

Fig. 2. SDS/15\%-polyacrylamide-gel electrophoresis of autolysed human $a$-thrombin

The analysis was performed under non-reducing conditions. Approx. $10 \mu \mathrm{g}$ portions of the samples were loaded after $24 \mathrm{~h}, 96 \mathrm{~h}$ and $144 \mathrm{~h}$ of autolysis. The fragments were detected by staining with Coomassie Blue. The chemical identity of each band was established (Table 1) after purification through gel filtration (Fig. 3) and h.p.l.c. (Fig. 4). Fragments A-B2 and A-B5 each contain two different molecules (Table 1) with only three amino acid residues difference at their $N$-terminal ends. Fragment A-B5 appeared on the gel as double bands (the two close bands in the middle at the $14000-M_{\mathrm{r}}$ region). 


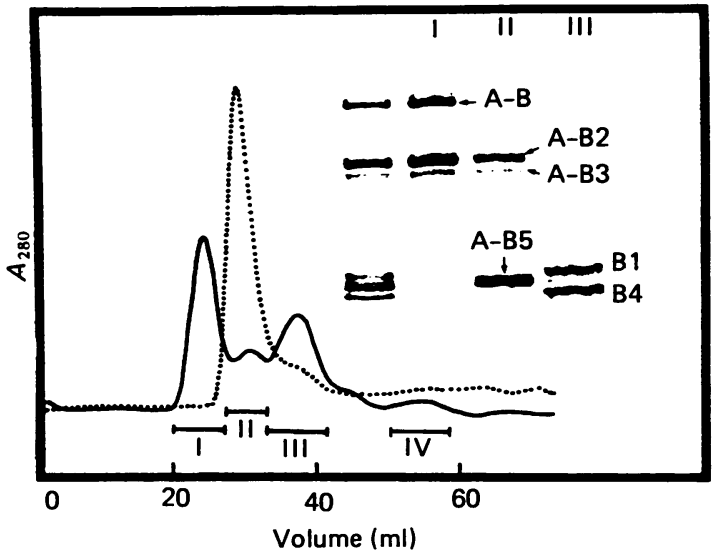

Fig. 3. Gel filtration (Sephadex G-75) of autolysed (144 h) human $a$-thrombin

The samples $(200 \mu \mathrm{g})$ were eluted with $50 \mathrm{~mm}$-ammonium bicarbonate $(\cdots \cdots)$ and $1 \mathrm{M}$-acetic acid ( -$)$. Four fractions were collected from the sample eluted with $1 \mathrm{M}$-acetic acid and fragments contained in each fraction were analysed by SDS $/ 15 \%$-polyacrylamide-gel electrophoresis (inset, the unlabelled gel track at the left side is the unfractionated sample). The size of column was $1.5 \mathrm{~cm} \times 30 \mathrm{~cm}$. The flow rate was $5 \mathrm{ml} / \mathrm{h}$.

very closely migrating bands. Component $\mathrm{B} 4$ represents a fragment containing residues 155-259 of the B-chain. Quantitative $N$-terminal analysis and sequence analysis, however, indicated that $17 \%$ of fragment B4 was cleaved at Lys-Ser (residues $212-213$ ) and $10 \%$ of fragment B4 was cleaved at Arg-Ile (residues 180-181). However, these further cleaved fragments were held together by two disulphide linkages located within fragment B4 (connecting residue 173 with 187 and residue 201 with 231).

Fig. 3 shows chemical features of the autolysed human $\alpha$-thrombin. When $200 \mu \mathrm{g}$ of the $144 \mathrm{~h}$-autolysed $\alpha$ thrombin was separated on a Sephadex G-75 column with $50 \mathrm{~mm}$-ammonium bicarbonate as eluent, both $\alpha$-thrombin and the autolysed derivatives were eluted together (Fig. 3, dotted curve), an indication that non-covalent binding holds these autolysed fragments together in alkaline solution. When the same sample was separated on the same column with $1 \mathrm{M}$-acetic acid as eluent, three fractions were obtained (Fig. 3, continuous curve). SDS/polyacrylamide-gel analysis (Fig. 3, inset) indicated that fraction I contained fragments A-B, A-B2 and A-B3, fraction II contained mainly fragment A-B5 with minor amounts of fragments $A-B 2$ and $A-B 3$, and fraction III contained fragments B1 and B4. Components in each of these three fractions were further purified by using the h.p.l.c. conditions described in Fig. 4. A fourth fraction in Fig. 3 was also collected. Quantitative $N$-terminal analysis and peptide analysis (results not shown) indicated that this fraction contained at least 30 different peptides with the amounts constituting less than $10-15 \%$ of the main autolysed products.

When amounts of fragments A-B, A-B2, A-B3 and A-B5 were taken as the measurement of $\alpha-, \beta-, \beta^{\prime}$ - and $\gamma$-thrombins respectively (Fig. 5), the rate of $\alpha$-thrombin autolysis and the appearance of $\beta$-, $\beta^{\prime}$ - and $\gamma$-thrombins can be quantitatively analysed by using the h.p.l.c. system described in Fig. 4. A $144 \mathrm{~h}$-autolysed human $\alpha$-thrombin contains (in $\mathrm{mol} \%$ ) $18 \%$ of $\alpha$-thrombin derivative, $24 \%$ of $\beta$-thrombin derivative, $9 \%$ of $\beta^{\prime}$-thrombin derivative and $22 \%$ of $\gamma$-thrombin derivative. These derivatives add up to only $73 \%$ of the total

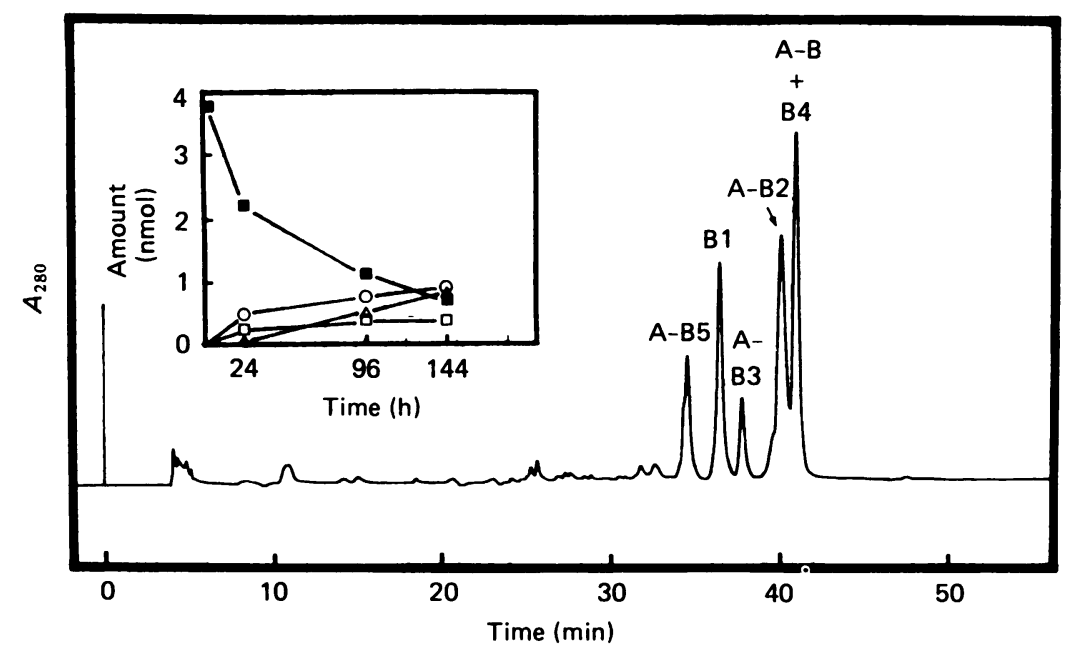

Fig. 4. H.p.l.c. separation of autolysed (144 h) human a-thrombin

Fractions, I, II and III from Fig. 3 can be further separated by this system to obtain each fragment derived from $\beta$-, $\beta^{\prime}$ - and $\gamma$-thrombins in pure form. The intact $\alpha$-thrombin (A-B) was co-eluted with fragment B4. The quantity and identity of each fragment were determined by amino acid analysis and sequence analysis. The decrease of $\alpha$-thrombin (A-B, $\square$ ) and the appearance of $\beta$-thrombin (A-B2, O) $\beta^{\prime}$-thrombin (A-B3, $\square$ ) and $\gamma$-thrombin (A-B5, $\triangle$ ) can be quantitatively analysed (inset). Chromatography was on a column of Vydac $C_{18}$ (218TP54); the column temperature was $25^{\circ} \mathrm{C}$. Solvent $\mathrm{A}$ was $0.5 \%$ (v/v) trifluoroacetic acid in water, and solvent $\mathrm{B}$ was $0.5 \%(\mathrm{v} / \mathrm{v})$ trifluoroacetic acid in acetonitrile; the gradient was $10 \%$ solvent $\mathrm{B}$ to $50 \%$ solvent B in $\mathbf{4 0}$ min, kept at $50 \%$ solvent B until 50 min and returned to $10 \%$ solvent $\mathrm{B}$ in $10 \mathrm{~min}$. The chart speed was $20 \mathrm{~cm} / \mathrm{h}$. 
Table 1. Structures of fragments derived from the autolysis of human $a$-thrombin

For the nomenclature and $M_{\mathrm{r}}$ value of each fragment, refer to Fig. 2 . The $N$-terminal sequences and structures are supported by the data for $M_{\mathrm{r}}$ values (Fig. 2), $N$-terminal sequencing and amino acid analysis. The $C$-terminal sequences were not performed.

Fragment

$N$-Terminal sequences and structures§

A-B

A-B2

Native human $\alpha$-thrombin is composed of A-chain (36 amino acid residues) and

B-chain (259 amino acid residues) linked together through a disulphate bond

(A) TFGSGEAD-GL-- (intact A-chain)

(B2) * ( X ERNIEKISM - - (residues 71-259 of the B-chain, about $54 \%$ )

N IEXIS M E K I - - (residues 74-259 of the B-chain, about $46 \%$ )

A-B3

(A) TFGSGEAD-GI-- - (intact A-chain)

A-B5

(B3) IVEG S DAEIGM - - - (residues 1-154 of the B-chain)

(A) TFGSGEAD-GL-- - (intact A-chain)

$\{$ (B5) * Y Y RNIEKI S M - - - (residues 71-154 of the B-chain, about 52\%)

N IE K I S M E K I - - (residues 74-154 of the B-chain, about $48 \%$ )

B1

IVE G D A I G M S - (mixtures of residues 1-70 and 1-73 of the B-chain)

B4

G QP S V L QVVNL P - - (residues 155-259 of the B-chain)

* B2 and B5 are two species of molecules resulting from major autolytic cleavages at residues 70-71 (Arg-Tyr) and residues 73-74 (Arg-Asn).

$\alpha$-thrombin. The difference is most likely due to further fragmentation of $\beta-, \beta^{\prime}$ - and $\gamma$-thrombins and their remains can be found in the fraction IV in Fig. 3.

\section{Proteolytic specificities of autolysed human $a$-thrombin}

The proteolytic specificities of autolysed human $\alpha$-thrombin are summarized in Table 2. A $144 \mathrm{~h}$ autolysed sample with only $18 \%$ of the remaining $\alpha$-thrombin was selected for study. Autolysed $\alpha$-thrombin lost more than $99 \%$ of its clotting activity, but retained the same specificity and substantial catalytic activity towards tripeptide substrates and non-fibrinogen polypeptide hormones and proteins (Table 2). These non-fibrinogen polypeptides have been shown to be selectively cleaved by $\alpha$-thrombin at one major Arg-Xaa bond (Chang, 1985) (indicated by arrows in Table 2).

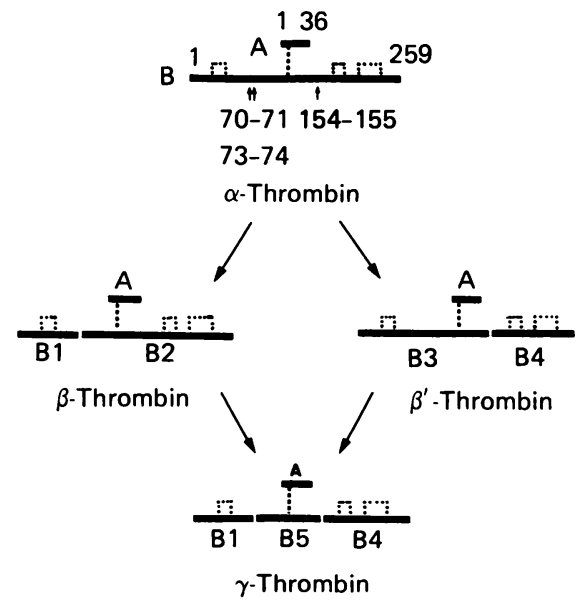

Fig. 5. Major autolysis pathway of human a-thrombin

The detailed structure of each fragment is listed in Table 1. It is relevant to point out that this major pathway is accompanied by minor and random cleavages, which prevail after approx. $144 \mathrm{~h}$ of autolysis and lead to the eventual breakdown of all forms of thrombin.
Further experiments (results not shown) also demonstrated that both native $\alpha$-thrombin and $144 \mathrm{~h}$-autolysed sample cleaved the Pro-Arg-Thr bond of eel calcitonin at a much lower efficiency than that of salmon and glycine amide-modified eel calcitonins (Chang, 1985). This suggests that an acidic residue at $\mathbf{P} 2^{\prime}$ position critically hinders the catalytic efficiency of $\alpha$-thrombin as well as $\beta$-, $\beta^{\prime}$ - and $\gamma$-thrombins.

\section{DISCUSSION}

There are two questions relating to the $\alpha$-thrombin autolysis that are of particular interest. The first pertains to the pathway of $\alpha$-thrombin autolysis. In particular, which peptide bonds are cleaved during autolysis and what are their relative cleavage rates? Secondly, it is known that autolysed $\alpha$-thrombin loses its clotting activity but retains substantial proteolytic activity towards small polypeptide substrates (Fenton et al., 1977b, 1978; Lundblad et al., 1979). Can these functional differences be explained, at the molecular level, by the structural differences between $\alpha$-thrombin and autolysed $\alpha$-thrombin? Earlier work by Fenton $e t$ al. (1977a) has shown that autolytic cleavage of human $\alpha$-thrombin at positions 73-74 of the B-chain produced $\beta$-thrombin, whereas subsequent cleavage at positions 154-155 gave rise to the $\gamma$-thrombin. A report by Boissel et al. (1984) pointed out that conversion of human $\alpha$-thrombin into $\beta$-thrombin resulted from two cleavages at Arg-62 and Arg-73 in the B-chain, releasing an intact II-residue peptide (Fig. 6). Conversion of $\beta$-thrombin into $\gamma$-thrombin corresponded to the additional loss of a fragment of the B-chain cleaved at Arg-123 and Lys-154. Similar cleavage sites were observed for conversion of bovine $\alpha$-thrombin into $\beta$-thrombin (Fig. 6) (Lundblad et al., 1979). However, no data are available from this earlier work to show the relative rate of autolysis at each site.

In the present study I found that $\alpha$-thrombin undergoes autolytic cleavage at Arg-Tyr (residues 70-71), which has not been previously reported. The combined cleavage rate of Arg-Tyr (residues 70-71) and 
Table 2. Proteolytic activities of a 144 h-autolysed human a-thrombin

The autolysed sample contains $18 \%$ of a $\alpha$-thrombin, $24 \%$ of $\beta$-thrombin, $9 \%$ of $\beta^{\prime}$-thrombinand $22 \%$ of $\gamma$-thrombin. The initial rates were calculated and are given as percentages based on those from the native $\alpha$-thrombin taken as $100 \%$.

\begin{tabular}{|c|c|c|}
\hline Substrate & Specific cleavage site $(\boldsymbol{l})$ & $\begin{array}{c}\text { Initial } \\
\text { rate }(\%)\end{array}$ \\
\hline Human fibrinogen & Specific release of fibrinopeptides A and B & 20 \\
\hline Chromozym TH & Tosy1-G-P-R-4-nitroan111de & 94 \\
\hline Antibody $\kappa$-chain & 1 T T PRTFGG & 70 \\
\hline Gastrin-releasing peptide & APVSVGGGTVLAKMYPR'GNHWAVGBLM & 79 \\
\hline Salmon calcitonin & CSNLSTCVLGXLSQELHKLQTYPRTNTGSGTP & 85 \\
\hline Eel calcitonin (Gly- $\mathrm{NH}_{2}$-modified) & CSNLSTCVLGKLSQELHKLQTYPRTDVGAGTP & 88 \\
\hline$A \alpha(1-23)$ & ADSGEGDFLAEGGGVR'GRVVER & 55 \\
\hline Human B-chain & $\stackrel{60}{60 u-L e u-V a l-A r g-\text { Ile-Gly-Lys-His-Ser-Arg-Thr-Arg-Tyr-Glu-Arg-Asn }}$ & \\
\hline Bovine B-chain & $\begin{array}{c}60 \\
\text { Leu-Leu-Val-Arg-Ile-Gly-Lys-His-Ser-Arg-Thr-Arg-Tyr-Glu-Arg-Lys }\end{array}$ & \\
\hline
\end{tabular}

Fig. 6. Autolytic sites of human and bovine $a$-thrombins leading to formation of the $\beta$-thrombins

In the bovine $\alpha$-thrombin, Lys-His and Arg-Lys were found to be cleaved during the conversion of $\alpha$-thrombin into $\beta$-thrombin (Lundblad et al., 1979). In the human $\alpha$-thrombin, Arg-Ile and Arg-Asn were found to be autolytically cleaved during the conversion of $\alpha$-thrombin into $\beta$-thrombin (Fenton et al., 1977a; Boissel et al., 1984). In the present study Arg-Tyr and Arg-Asn cleavages were detected.

Arg-Asn (residues 73-74) (see Fig. 1), which generates $\beta$-thrombin, is faster than that of Lys-Gly. However, there is no apparent lag phase for the cleavage of Lys-Gly (residues 154-155), as would be expected if it depended on a prior cleavage of Arg-Asn or Arg-Tyr. Existence of a

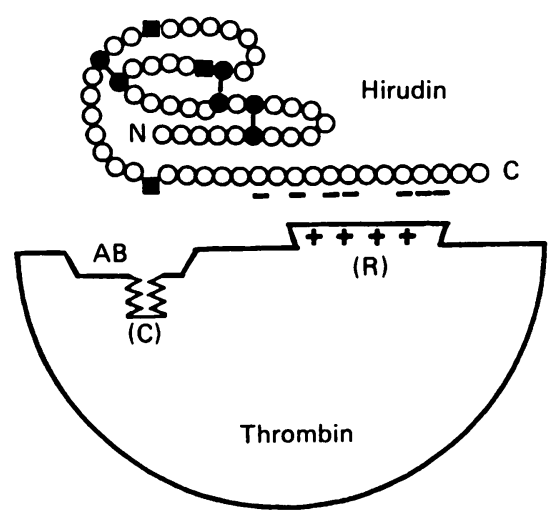

Fig. 7. Proposed model for the multiple functional sites of human a-thrombin

Human $\alpha$-thrombin contains a constrained catalytic site (C), an apolar binding site (AB) and a recognition site (R), which is comprised of basic amino acid residues including those located within residues 62-73 of the B-chain. Binding of this basic recognition site to the acidic $C$-terminal segment of hirudin produces an exceedingly stable $\alpha$-thrombin-hirudin complex. The negatively charged residues in the $C$-terminal region of hirudin are marked. Three disulphide linkages (-O) (Dodt et al., $1985)$ and three lysine residues $(\square)$ in hirudin are also indicated. new form of thrombin derivative (designated as $\beta^{\prime}$-thrombin) with cleavage at Lys-Gly alone was confirmed by the isolation of fragment A-B3 (Figs. 2 and 4). Furthermore, with the $144 \mathrm{~h}$-autolysed sample, I have not been able to detect significant cleavages at Arg-62 and Arg-123, which were observed previously (Boissel et al., 1984). This discrepancy may be due to the different conditions of autolysis employed. Boissel et al. (1984) prepared the autolysed thrombin with the conditions of $2.4-4 \mathrm{mg} / \mathrm{ml}$ in $0.02 \mathrm{M}-\mathrm{NaCl}$ solution, $\mathrm{pH} 8.6$, at $20^{\circ} \mathrm{C}$. I used the concentration of $1 \mathrm{mg} / \mathrm{ml}$ in $50 \mathrm{~mm}$ ammonium bicarbonate solution, $\mathrm{pH} 8.0$, at $25^{\circ} \mathrm{C}$. I also employed a different approach in obtaining structural information on autolysed thrombin. In addition to the analysis of purified fragments, the structure of autolysed thrombin was first evaluated by quantitative $N$-terminal analysis before proceeding to any chromatographic purification. This approach allows the rates of three major autolytic cleavages to be followed quantitatively and facilitates the observation of many minor autolytic sites. So far, in both bovine and human $\alpha$-thrombins, at least five Arg/Lys-Xaa bonds within the region 62-73 in the B-chain have been shown to be susceptible to autolysis (Fig. 6). This interesting fact implies that $\mathrm{Arg} / \mathrm{Lys}$ bonds within residues $62-73$ of the B-chain may be clustered on the surface of the $\alpha$-thrombin molecule and may play a role in both fibrinogen and hirudin recognition (Figs. 6 and 7) (Chang, 1983a).

Analysis of the proteolytic specificity of autolysed $\alpha$-thrombin provides further insight into the structure and function relationship of human $\alpha$-thrombin. Lundblad et al. (1984) have suggested that the differences in the fibrinogen clotting activity of bovine $\alpha$ - and $\beta$-thrombins can result from changes in the reactivity of the active-site histidine residue. In this study, the activity 
of autolysed thrombin (mixture of $\beta-, \beta^{\prime}$ - and $\gamma-$ thrombins) was measured against various sizes of non-fibrinogen polypeptide substrates. The experiment reveals that autolysed human thrombin retains the same specificity and substantial proteolytic activity towards many non-fibrinogen peptides and proteins (Table 2). These results suggest that mechanism for the selective cleavages of fibrinogen and non-fibrinogen substrates are controlled at two different levels. Autolysis has minimal effect on the active site of human thrombin that governs the selective cleavages of non-fibrinogen substrates. However, autolysis disrupts a structural element (recognition site) that is essential for the efficient release of fibrinopeptides from fibrinogen. This recognition site is most likely an Arg/Lys cluster situated at the major autolytic sites of human $\alpha$-thrombin. Fig. 7 shows a proposed model of human $\alpha$-thrombin with multiple functional sites. $\alpha$-Thrombin contains a constrained catalytic site (Fenton et al., 1978; Chang, 1985), an apolar binding site extending towards both $\mathrm{N}$ - and $C$-terminal directions of the catalytic site (Berliner \& Shen, 1977; Sonder \& Fenton, 1984; Chang, 1985) and an independent recognition site that is composed of clusters of Arg and Lys residues from the 62-73 region of the B-chain (Chang, 1983a; Berliner et al., 1985). Binding of this basic recognition site to the acidic $C$-terminal segment of hirudin produces a highly stable $\alpha$-thrombin-hirudin complex (Markwardt, 1970; Fenton et al., 1978; Chang, 1983a). Binding of this basic recognition site to the acidic fibrinopeptides A and B (Chang, 1983a) resulted in efficient cleavages of fibrinopeptides A and B from fibrinogen. Disruption of this recognition site by autolysis, trypsin digestion (Fenton et al., 1978) or chemical modification (Landaburu \& Seegers, 1960; Magnusson, 1972) impairs the clotting activity and hirudin-binding affinity of $\alpha$-thrombin.

I am indebted to Dr. J. W. Fenton, II, for his generosity of providing human $\alpha$-thrombin for this study. I also acknowledge discussions with Dr. P. Braun, Dr. J. Hofsteenge, Dr. J. Lucas, Dr. A. Matus and Dr. V. Steiner during the preparation of this manuscript. I am also grateful to $\mathrm{Mr}$. R. Knecht and W. Segmuller for performing the automatic sequence determination.

\section{REFERENCES}

Alkan, S. S., Ball, R., Chang, J.-Y. \& Braun D. G. (1983) Molecular Immunol. 20, 203-211

Berliner, L. J. \& Shen, Y. Y. L. (1977) Biochemistry 16, $4622-4626$

Received 24 March 1986/12 June 1986; accepted 26 August 1986
Berliner, L. J., Sugawara, Y. \& Fenton, J. W., II (1985) Biochemistry 24, 7005-7009

Blombäck, B., Blombäck, M., Hessel, B. \& Iwanagan, S. (1967) Nature (London) 215, 1445-1448

Boissel, J.-P., Bonniec, B. L., Rabiet, M.-J., Labie, D. \& Elion, J. (1984) J. Biol. Chem. 259, 5691-5697

Chang, J.-Y. (1983a) FEBS Lett. 164, 307-313

Chang, J.-Y. (1983b) Methods Enzymol. 91, 79-84

Chang, J.-Y. (1983c) Methods Enzymol. 91, 455-466

Chang, J.-Y. (1985) Eur. J. Biochem. 151, 217-224

Chang, J.-Y., Knecht, R. \& Braun, D. G. (1983) Methods Enzymol. 91, 41-48

Chang, J.-Y., Alkan, S. S., Hilschmann, N. \& Braun, D. G. (1985) Eur. J. Biochem. 151, 225-230

Dodt, J., Seemuller, U., Maschler, R. \& Fritz, H. (1985) Biol. Chem. (Hoppe-Seyler) 366, 379-385

Fenton, J. W., II (1981) Ann. N.Y. Acad. Sci. 370, 468-495

Fenton, J. W., II, Landis, B. H., Walz, D. A. \& Finlayson, J. S. $(1977 a)$ in Chemistry and Biology of Thrombin (Lundblad, R. L., Fenton, J. W., II \& Mann, K. G., eds.), pp. 43-70, Ann Arbor Scientific Publishers, Ann Arbor

Fenton, J. W., II, Fasco, M. J., Stackrow, A. B., Aronson, D. L., Young, A. M. \& Finlayson, J. S. (1977b) J. Biol. Chem. 252, 3587-3598

Fenton, J. W., II, Landis, B. H., Walz, D. A., Bing, D. H., Feinmann, R. D., Zabinski, M. P., Sonder, S. A., Berliner, L. J. \& Finlayson, J. S. (1978) in The Chemistry and Physiology of Human Plasma Proteins (Bing, D. H., ed.), pp. 151-183, Pergamon Press, Oxford

Glover, G. \& Shaw, E. (1971) J. Biol. Chem. 246, 4594-4601

Gorman, J. J., Gastaldi, P. A. \& Shaw, E. (1976) Biochem. Biophys. Acta 439, 1-16

Hanna, L. S., Scheraga, H. A., Francis, C. W. \& Marder, V. (1984) Biochemistry 23, 4681-4687

Hogg, D. H. \& Blombäck, B. (1978) Thromb. Res. 12, 953-964

Hunkapiller, M. W., Hewick, R. M., Dreyer, W. J. \& Hood, L. E. (1983) Methods Enzymol. 91, 399-412

Knecht, R., Seemuller, U., Liersch, M., Fritz, F., Braun, D. G. \& Chang, J.-Y. (1983) Anal. Biochem. 130, 65-71

Laemmli, U. K. (1970) Nature (London) 227, 680-686

Landaburu, R. H. \& Seegers, W. H. (1960) Can. J. Biochem. Biophys. 38, 613-619

Lundblad, R. L., Kingdon, H. S. \& Mann, K. G. (1976) Methods Enzymol. 45, 156-176

Lundblad, R. L., Noyes, C. M., Mann, K. G. \& Kingdon, H. S. (1979) J. Biol. Chem. 254, 8524-8528

Lundblad, R. L., Nesheim, M. E., Straight, D. L., Sailor, S., Bowie, J., Jenzano, J. W., Roberts, J. D. \& Mann, K. G. (1984) J. Biol. Chem. 259, 6991-6995

Magnusson, S. (1972) Enzymes 3rd Ed. 3, 271-321

Markwardt, F. (1970) Methods Enzymol. 19, 924-932

Marsh, H. S., Meinwald, Y. C., Thannhauser, T. W. \& Scheraga, H. A. (1983) Biochemistry 22, 4170-4174

Rosenberg, R. D. \& Waugh, D. F. (1970) J. Biol. Chem. 245, 5049-5056

Sonder, S. A. \& Fenton, J. W., II (1984) Biochemistry 23, $1818-1823$ 